\title{
Long-term consequences of optical coherence tomography findings during percutaneous coronary intervention: the Centro Per La Lotta Contro L'infarto - Optimization Of Percutaneous Coronary Intervention (CLI-OPCI) LATE study
}

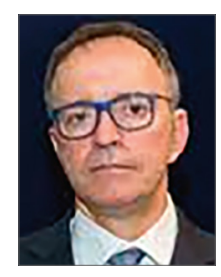

Francesco Prati ${ }^{1,2 *}, \mathrm{MD}$; Enrico Romagnoli1 ${ }^{1}, \mathrm{MD}, \mathrm{PhD}$; Alessio La Manna ${ }^{3}, \mathrm{MD}$; Francesco Burzotta ${ }^{4}, \mathrm{MD}$; Laura Gatto ${ }^{1}, \mathrm{MD}$; Valeria Marco $^{2}, \mathrm{RN}$; Massimo Fineschi ${ }^{5}, \mathrm{MD}$; Franco Fabbiocchi ${ }^{6}, \mathrm{MD}$; Francesco Versaci ${ }^{7}, \mathrm{MD}$; Carlo Trani ${ }^{4}, \mathrm{MD}$; Corrado Tamburino ${ }^{3}, \mathrm{MD}$; Fernando Alfonso ${ }^{8}, \mathrm{MD}$; Gary S. Mintz ${ }^{9}, \mathrm{MD}$; on behalf of CLI-OPCI Project Investigators

1. San Giovanni Addolorata Hospital, Rome, Italy; 2. Centro per la Lotta Contro L'Infarto - CLI Eoundation, Rome, Italy;

3. Division of Cardiology, University of Catania, Catania, Italy; 4. Università Cattolica del Sacro Cuore, Rome, Italy;

5. Azienda Ospedaliera Universitaria Senese, Siena, Italy; 6. Centro Cardiologico Monzino, IRCCS, Milan, Italy;

7. Santa Maria Goretti Hospital, Latina, Italy; 8. Hospital Universitario de La Princesa, Madrid, Spain; 9. Cardiovascular Research Foundation, New York, USA

\section{KEYWORDS}

- clinical research

- optical coherence

tomography

- risk stratification

\section{Abstract}

Aims: The role of intraprocedural optical coherence tomography (OCT) on the long-term clinical outcome of percutaneous coronary interventions (PCI) remains undefined. The aim of the present study was to evaluate the impact of quantitative OCT-defined suboptimal stent implantation at long-term follow-up.

Methods and results: In the context of the multicentre Centro per la Lotta contro l'Infarto - Optimisation of Percutaneous Coronary Intervention (CLI-OPCI) registry, we compared the long-term PCI outcome of 1,211 patients from 13 independent OCT-experienced centres according to end-procedural OCT findings. OCT assessment revealed suboptimal stent implantation in $30.9 \%$ of lesions, with an increased prevalence in patients experiencing device-oriented cardiovascular events (DoCE) (52.8\% vs. 28.0\%, p<0.001). At a median follow-up of 833 (interquartile range 415-1,447) days, in-stent minimum lumen area (MLA) $<4.5 \mathrm{~mm}^{2}$ (HR 1.82, $<<0.001$ ), distal stent edge dissection $>200 \mu \mathrm{m}$ (HR 2.03, $\left.\mathrm{p}=0.004\right)$, and significant reference vessel plaque and lumen area $<4.5 \mathrm{~mm}^{2}$ at either the distal (HR 5.22, $<<0.001$ ) or proximal (HR $5.67, \mathrm{p}<0.001)$ stent edges were independent predictors of device failure. Conversely, in-stent MLA/mean reference lumen area $<70 \%$, acute stent malapposition, and intra-stent plaque/thrombus protrusion were not associated with worse outcomes. Using multivariable Cox hazard analysis, the presence of at least one of the significant criteria for suboptimal OCT stent deployment was confirmed as an independent predictor of DoCE (HR 1.92, $\mathrm{p}=0.001)$.

Conclusions: Suboptimal stent deployment, defined according to specific quantitative OCT criteria, was confirmed as an independent outcome predictor at long-term follow-up.

\footnotetext{
*Corresponding author: Cardiology Unit, San Giovanni Addolorata Hospital, Via Amba Aradam 9, 00184 Rome, Italy. E-mail:fprati@hsangiovanni.roma.it
} 


\section{Abbreviations}

IVUS intravascular ultrasound

OCT optical coherence tomography

$\mathrm{PCl}$ percutaneous coronary intervention

DoCE device-oriented cardiovascular events

MLA minimum lumen area

SM stent malapposition

\section{Introduction}

The role of intravascular imaging guidance (intravascular ultrasound [IVUS] and optical coherence tomography [OCT]) to optimise percutaneous coronary interventions (PCI) is still much debated ${ }^{1-4}$. IVUS studies have shown that the two strongest and most consistent predictors of early stent thrombosis or restenosis after bare metal stent (BMS) or drug-eluting stent (DES) implantation are stent underexpansion (or a smaller intra-stent minimum lumen area [MLA] as a consequence of tissue/thrombus protrusion after stenting a culprit lesion in a patient presenting with an acute coronary syndrome $[\mathrm{ACS}]$ ) and geographic miss (including uncovered edge dissections $)^{5}$. These IVUS predictors of device-oriented cardiovascular events (DoCE) have been confirmed by OCT studies ${ }^{4,6,7}$.

In this context, the Centro per la Lotta Contro l'Infarto Optimisation of Percutaneous Coronary Intervention (CLI-OPCI) project was specifically designed to translate $\mathrm{OCT}$ findings into an effective clinical improvement. However, one limitation of previous IVUS and OCT reports - including the CLI-OPCI II study resided in the relatively short period of observation and the limited number of adverse events, leaving some uncertainties on the longterm clinical role of the metrics that were identified.

The aim of the present CLI-OPCI substudy (CLI-OPCI LATE) was to evaluate the impact of quantitative OCT-defined suboptimal stent implantation at long-term follow-up. In particular, we explored the predictive accuracy of the already validated criteria of suboptimal stent deployment in a large study population including 1,211 patients with a median follow-up duration of approximately three years.

Editorial, see page 370

\section{Methods}

\section{STUDY DESIGN AND ENDPOINTS}

The design and aims of the CLI-OPCI project have been described previously ${ }^{7,8}$. Briefly, the CLI-OPCI registry, aiming to assess the impact of OCT findings during PCI, was collected from participating centres (Appendix) who submitted data on all consecutive PCIs performed with the support of OCT imaging. Due to the exploratory nature of the project, only end-procedural OCT images were collected and included in the final analyses; indication for OCT, PCI technique, and additional manoeuvres derived from OCT use were left to each operator's choice. By protocol, all included cases had at least one good quality OCT pullback in the treated vessel that was carried out at the end of the procedure with a sufficient acquisition length to address the whole stented segment(s) and at least $5 \mathrm{~mm}$ of the adjacent reference segment(s $)^{1,9,10}$, with the exclusion of ostial lesions, in which proximal references were not assessed.
The correlation between end-procedural OCT-defined suboptimal stent deployment and long-term DoCE constituted the primary endpoint of this substudy; the impact of individual predictive quantitative OCT criteria previously validated was also evaluated. DoCE were a composite of cardiac mortality, target vessel myocardial infarction (MI; defined as CK-MB $>3$ times the upper limit of normal), and target lesion revascularisation (TLR) $)^{11}$. Outcomes were defined according to the Academic Research Consortium guidelines $^{12}$ and adjudicated blindly by a clinical events committee. The project was approved by the local ethics board and all patients provided written informed consent for the index procedure, phone/direct visit follow-up, and anonymous data management. This work was supported by the Centro per la Lotta Contro l'Infarto - Fondazione Onlus (Rome, Italy) and the authors were solely responsible for the design, conduct, and final content of this study.

\section{PROCEDURES AND IMAGING ACQUISITION}

Coronary angiography and PCIs were performed using standard techniques and catheters, according to the local common practice. OCT images was acquired by means of the $\mathrm{C} 7-\mathrm{XR}^{\mathrm{TM}}$ FD-OCT ${ }^{\text {TM }}$ Imaging System or the OPTIS ${ }^{\text {TM }}$ Imaging System (both St. Jude Medical, St. Paul, MN, USA) with a non-occlusive technique according to a well-standardised methodology 1,9,10 $^{10}$. Treatment choices (i.e., interventional technique and stent selection) were left to each operator's discretion, post-procedural dual antiplatelet therapy was recommended for at least twelve months, and patients were regularly followed by means of periodic phone calls and/or direct visit. In case of any adverse event or hospitalisation during the follow-up, additional visits were planned to obtain source documents and for proper adjudication of events.

\section{OCT ANALYSES AND DEFINITIONS}

All angiographic and OCT images were analysed in a blinded fashion off-line by expert readers at a central core laboratory (Rome Heart Research). OCT images were reconstructed using the Offline Review Workstation ILUMIEN ${ }^{\mathrm{TM}} / \mathrm{OPTIS}^{\mathrm{TM}}$ system (St. Jude Medical)', while dedicated automatic edge-detection software (Medis medical imaging systems bv, Leiden, the Netherlands) was adopted for quantitative coronary angiographic (QCA) assessment $^{13}$. Definitions for OCT were derived from available consensus documents ${ }^{1}$, and quantitative cut-offs were derived from the CLI-OPCI registries ${ }^{6,7}$ using the receiver operating characteristic (ROC) curve and highest Youden's index (J) to confirm the predictive accuracy and the maximum potential effectiveness ${ }^{14,15}$ :

- Malapposition: stent strut detachment from adjacent vessel wall $>200 \mu \mathrm{m}^{16,17}$;

- Edge dissection: linear rim of tissue $\geq 200 \mu \mathrm{m}$ in width and with a clear separation from the vessel wall or underlying plaque adjacent $(<5 \mathrm{~mm})$ to a stent edge $\mathrm{e}^{1,17}$;

- Reference lumen narrowing: lumen area $<4.5 \mathrm{~mm}^{2}$ in the presence of significant $(>70 \%)$ residual plaque adjacent to a stent edge $\mathrm{e}^{7,17}$;

- In-stent minimum lumen area (MLA): MLA $<4.5 \mathrm{~mm}^{2}$ assessed along the entire stent length ${ }^{7,17}$; 
- Residual stenosis: in-stent MLA $<70 \%$ of the average reference lumen $\operatorname{area}^{7,17}$;

- Eccentricity index: maximum stent diameter/minimum stent diameter ratio $<0.7$ at $\mathrm{MLA}^{17}$;

- Intra-stent plaque/thrombus protrusion: tissue prolapse $\geq 500 \mu \mathrm{m}$ in thickness among stent struts into the vessel lumen ${ }^{16,18}$

Suboptimal OCT stent deployment required the presence of at least one of these OCT findings significantly associated with DoCE.

\section{STATISTICAL ANALYSIS}

Mean ( \pm standard deviation) or median $\left(1^{\text {st }}-3^{\text {rd }}\right.$ quartile $)$ was used to describe continuous variables in case of normal or skewed distribution, respectively; percentages were used to report discrete variables. The Student's t-test, Mann-Whitney U test, $\chi^{2}$ test, and Fisher's exact test were applied for bivariate analyses when appropriate. DoCE were evaluated on a per-patient hierarchical basis, compared with the log-rank test, and summarised as Kaplan-Meier estimates. A landmark analysis at one year was also performed to provide separate descriptions of the midterm and late events. TLR and stent thrombosis were analysed on both a per-patient and a per-lesion basis, and a generalised mixed model analysis was performed to exclude differences due to lesion and patient level clustering. All variables reported in Table 1, Table 2 and Table 3 were tested for bivariate association with DoCE and, if nominally significant $(\mathrm{p}<0.05)$, were simultaneously forced into a Cox regression model to identify independent outcome predictors and to calculate their adjusted hazard ratios (HRs). The final Cox regression model included the following variables: age, left ventricular ejection fraction (LVEF), diabetes mellitus, chronic kidney disease, family history of coronary artery disease (CAD), prior MI, angiographically ambiguous lesion (i.e., intermediate lesion with irregular contour and/or haziness), ostial lesion treatment, bare metal stent (BMS) or bioabsorbable vascular scaffold (BVS) usage, and suboptimal final OCT result. Statistical analyses were carried out using SPSS Predictive Analytics Software (PASW), Version 22.0 (IBM Corp., Armonk, NY, USA) and adopting a two-tailed $\mathrm{p}$-value $<0.05$ for statistical significance.

\section{Results}

Between 2009 and 2013, 1,211 patients with 1,422 lesions undergoing end-procedural OCT assessment were enrolled in this registry from 13 independent OCT-experienced centres. Table 1 and Table 2 summarise the clinical and procedural characteristics of the study population. Median patient age was 64 (interquartile range [IQR] 56-72) years, with $20.8 \%$ females. The prevalence of diabetes mellitus and chronic kidney disease was $21.6 \%$ and $13.6 \%$, respectively. A history of previous MI was present in $21.4 \%$ of cases, previous coronary revascularisation in $30.9 \%$, and about half of the patients had multivessel disease. An ACS was the admission diagnosis in $58.2 \%$ of patients, including acute MI in $41.6 \%$.

Treated lesions usually had a complex profile (Ellis Class B2/C, $78.2 \%$ ), predilation was performed in $73.0 \%$ of lesions, high-pressure stent post-dilatation in $55.8 \%$, DES implantation in $73.1 \%$, and multiple overlapping stents in $21.1 \%$. A satisfactory angiographic result (residual stenosis $<30 \%$ with TIMI 3 flow) was obtained in $97.7 \%$ of treated lesions with $2.9 \%$ periprocedural MI.

End-procedural OCT assessment disclosed an in-stent MLA $<4.5 \mathrm{~mm}^{2}$ in $23.8 \%$ with asymmetry in $6.4 \%$ of the stented lesions,

Table 1. Patient characteristics.

\begin{tabular}{|c|c|c|c|c|}
\hline & $\begin{array}{l}\text { Total population } \\
(1,211)\end{array}$ & $\begin{array}{c}\text { Patients with DoCE } \\
\text { (144) }\end{array}$ & $\begin{array}{l}\text { Patients without DoCE } \\
\qquad(1,067)\end{array}$ & $p$-value \\
\hline Age (years)* & $64(56-72)$ & $66(57-75)$ & $64(56-72)$ & 0.020 \\
\hline Female gender (\%) & $252(20.8)$ & $28(19.4)$ & $224(21.0)$ & 0.743 \\
\hline Left ventricle ejection fraction $(\%)^{*}$ & $55(48-60)$ & $54(45-60)$ & $55(48-60)$ & 0.003 \\
\hline Hypertension (\%) & $837(69.1)$ & 105 (72.9) & $732(68.6)$ & 0.281 \\
\hline Hypercholesterolaemia (\%) & $734(60.6)$ & $87(60.4)$ & $647(60.6)$ & 0.974 \\
\hline Smoking habit (\%) & $397(32.8)$ & $44(30.6)$ & $353(33.1)$ & 0.634 \\
\hline Family history of CAD (\%) & $378(31.2)$ & $32(22.2)$ & $346(32.4)$ & 0.016 \\
\hline Diabetes mellitus (\%) & $262(21.6)$ & 49 (34.0) & $213(20.0)$ & $<0.001$ \\
\hline CKD (GFR <60 ml/min/1.73 m²) (\%) & $165(13.6)$ & $29(20.1)$ & $136(12.7)$ & 0.011 \\
\hline Multivessel disease $(\%)$ & $646(53.3)$ & $86(59.7)$ & $560(52.5)$ & 0.097 \\
\hline Prior MI (\%) & $259(21.4)$ & $45(31.3)$ & $214(20.1)$ & 0.003 \\
\hline Prior coronary revascularisation (\%) & $374(30.9)$ & $54(37.5)$ & $320(30.0)$ & 0.084 \\
\hline Acute coronary syndrome (\%) & $705(58.2)$ & $87(60.4)$ & $618(57.9)$ & 0.718 \\
\hline STEMI (\%) & $341(28.2)$ & $40(27.8)$ & $301(28.2)$ & 0.922 \\
\hline NSTEMI (\%) & $162(13.4)$ & $23(16.0)$ & $139(13.0)$ & 0.363 \\
\hline Unstable angina (\%) & $201(16.6)$ & $24(16.7)$ & $177(16.6)$ & 0.981 \\
\hline Stable angina (\%) & $496(41.0)$ & $57(39.6)$ & $439(41.1)$ & 0.718 \\
\hline
\end{tabular}

* Expressed as median and interquartile range. CAD: coronary artery disease; CKD: chronic kidney disease; DoCE: device-oriented cardiovascular events; MI: myocardial infarction; NSTEMI: non-ST-elevation MI; STEMI: ST-elevation MI 
Table 2. Procedural characteristics.

\begin{tabular}{|c|c|c|c|c|}
\hline & $\begin{array}{c}\text { All lesions } \\
(1,422)\end{array}$ & $\begin{array}{c}\text { Lesions with DoCE } \\
\text { (178) }\end{array}$ & $\begin{array}{l}\text { Lesions without DoCE } \\
\qquad(1,244)\end{array}$ & $p$-value \\
\hline \multicolumn{5}{|l|}{ Location of lesion treated } \\
\hline Left main $(\%)$ & $78(5.5)$ & $12(6.7)$ & $66(5.3)$ & 0.541 \\
\hline Left anterior descending artery (\%) & $763(53.6)$ & $90(50.6)$ & $673(54.1)$ & 0.421 \\
\hline Left circumflex artery $(\%)$ & $263(18.5)$ & $41(23.0)$ & $222(17.8)$ & 0.118 \\
\hline Right coronary artery (\%) & $318(22.4)$ & $35(19.7)$ & $283(22.8)$ & 0.408 \\
\hline \multicolumn{5}{|l|}{ Lesion features } \\
\hline Ellis Class B2/C (\%) & $1,112(78.2)$ & $143(80.3)$ & 969 (77.9) & 0.521 \\
\hline Calcific lesion (\%) & $207(14.6)$ & $32(18.0)$ & $175(14.1)$ & 0.199 \\
\hline Ostial lesion (\%) & $93(6.5)$ & $18(10.1)$ & $75(6.0)$ & 0.046 \\
\hline Bifurcation lesion (\%) & $220(15.5)$ & $28(15.7)$ & $192(15.4)$ & 0.985 \\
\hline Chronic total occlusion lesion (\%) & $34(2.4)$ & $4(2.2)$ & $30(2.4)$ & 0.939 \\
\hline Angiographically ambiguous lesion (\%) & $141(9.9)$ & $26(14.6)$ & $115(9.2)$ & 0.017 \\
\hline In-stent restenosis lesion (\%) & $84(5.9)$ & $16(9.0)$ & $68(5.5)$ & 0.104 \\
\hline Stent thrombosis lesion (\%) & $43(3.0)$ & $7(3.9)$ & $36(2.9)$ & 0.601 \\
\hline \multicolumn{5}{|l|}{ Technical approach } \\
\hline Direct stenting (\%) & $384(27.0)$ & $49(27.5)$ & 335 (26.9) & 0.872 \\
\hline Thrombectomy use (\%) & $194(13.6)$ & $23(12.9)$ & $171(13.7)$ & 0.915 \\
\hline Post-dilation (\%) & $794(55.8)$ & $91(51.1)$ & $703(56.5)$ & 0.254 \\
\hline DES (\%) & $1,040(73.1)$ & $121(68.0)$ & 919 (73.9) & 0.116 \\
\hline BMS (\%) & $232(16.3)$ & $48(27.0)$ & $184(14.8)$ & $<0.001$ \\
\hline BVS (\%) & $147(10.3)$ & $9(5.1)$ & $138(11.1)$ & 0.019 \\
\hline Overlapping stent (\%) & $300(21.1)$ & $40(22.5)$ & $260(20.9)$ & 0.710 \\
\hline Optimal angiographic result (\%) & $1,390(97.7)$ & $172(96.6)$ & $1,218(97.9)$ & 0.419 \\
\hline Stent diameter $(\mathrm{mm})^{*}$ & $3.0(2.75-3.5)$ & $3.0(2.5-3.25)$ & $3.0(2.75-3.5)$ & 0.057 \\
\hline Stent length $(\mathrm{mm}) *$ & $20(15-28)$ & $18(15-28)$ & $20(15-30)$ & 0.035 \\
\hline Max pressure during stent implantation * & $16(14-18)$ & $16(14-18)$ & $16(14-18)$ & 0.520 \\
\hline Contrast dye * & $250(200-300)$ & $250(200-350)$ & $250(200-300)$ & 0.173 \\
\hline
\end{tabular}

stent underexpansion in $22.4 \%$, edge dissection in $12.0 \%$, acute residual stent malapposition (SM) in $52.3 \%$, intra-stent plaque/thrombus protrusion in $27.5 \%$, and reference lumen narrowing in $6.3 \%$.

During the observed median follow-up of 833 (IQR 415-1,447) days, $11.9 \%$ of the patients experienced a DoCE including $3.4 \%$ cardiac mortality, $4.3 \%$ non-fatal target vessel MI, and $8.6 \%$ TLR (Table 4). Excluding periprocedural MI, mean time-to-DoCE was 754 (IQR range 376-1,383) days, with $55.6 \%$ of adverse events occurring within the first twelve months after the procedure and $44.4 \%$ occurring after twelve months. The rate of patients lost after the first follow-up contact (usually 30-180 days after PCI) was $6.8 \%$. Only one case of premature dual antiplatelet therapy (DAPT) discontinuation was documented in these cases. DAPT regimens included clopidogrel in $68.7 \%$, prasugrel in $19.2 \%$, and ticagrelor in $12.1 \%$ of patients, with no significant difference between patients with vs. those without adverse events.

\section{CLINICAL PREDICTORS}

When compared to patients with event-free survival, patients experiencing DoCE during follow-up showed a higher baseline risk profile including older age (66 vs. 64 years, $\mathrm{p}=0.020$ ), lower median LVEF ( $54 \%$ vs. $55 \%, \mathrm{p}=0.003$ ), a higher prevalence of diabetes mellitus $(34.0 \%$ vs. $20.0 \%, \mathrm{p}<0.001)$ and chronic kidney disease $(20.1 \%$ vs. $12.7 \%, p=0.011)$, and a more frequent history of prior MI (31.3\% vs. $20.1 \%, \mathrm{p}=0.003)$ (Table 1$)$.

Regarding procedural characteristics, patients with DoCE were characterised by higher BMS use $(27.0 \%$ vs. $14.8 \%, \mathrm{p}<0.001)$ and more frequent treatment of an ostial lesion (10.1\% vs. $6.0 \%$, $\mathrm{p}=0.046)$, or an angiographically ambiguous lesion $(14.6 \%$ vs. 9.2\%, $\mathrm{p}=0.017$ ) (Table 2).

In lesions associated with any adverse event during follow-up, OCT analyses revealed a significantly higher prevalence of suboptimal stent deployment in terms of in-stent MLA $<4.5 \mathrm{~mm}^{2}(35.4 \%$ vs. $22.1 \%, \mathrm{p}<0.001)$, dissection $>200 \mu \mathrm{m}$ at the distal stent edge $(10.7 \%$ vs. $5.6 \%, \mathrm{p}=0.007)$, and reference lumen area $<4.5 \mathrm{~mm}^{2}$ in the presence of residual significant plaque at either the distal $(15.7 \%$ vs. $3.1 \%, \mathrm{p}<0.001)$ or proximal $(8.4 \%$ vs. $1.1 \%, \mathrm{p}<0.001)$ stent edge (Figure 1). Conversely, other parameters such as eccentric stent expansion at the MLA site $(12.4 \%$ vs. $10.3 \%, p=0.479)$, in-stent MLA $<70 \%$ of the average reference lumen area $(25.8 \%$ vs. $21.9 \%$, $\mathrm{p}=0.208)$, dissection at the proximal stent edge $(7.9 \%$ vs. $6.3 \%$, $\mathrm{p}=0.518)$, acute $\mathrm{SM}>200 \mu \mathrm{m}(48.9 \%$ vs. $52.7 \%, \mathrm{p}=0.337)$, or in-stent 
Table 3. OCT findings.

\begin{tabular}{|c|c|c|c|c|}
\hline & $\begin{array}{c}\text { All lesions } \\
(1,422)\end{array}$ & $\begin{array}{c}\text { Lesions with DoCE } \\
\text { (178) }\end{array}$ & $\begin{array}{c}\text { Lesions without DoCE } \\
(1,244)\end{array}$ & $p$-value \\
\hline \multicolumn{5}{|l|}{ OCT features } \\
\hline Minimum in-stent lumen area $\left(\mathrm{mm}^{2}\right)$ & $6.0 \pm 2.1$ & $5.6 \pm 2.0$ & $6.1 \pm 2.1$ & 0.002 \\
\hline Maximum in-stent lumen diameter ( $\mathrm{mm}$ ) & $3.0 \pm 0.6$ & $2.9 \pm 0.5$ & $3.0 \pm 0.6$ & 0.006 \\
\hline Minimum in-stent lumen diameter (mm) & $2.4 \pm 0.5$ & $2.3 \pm 0.5$ & $2.4 \pm 0.5$ & 0.006 \\
\hline Lumen symmetry (\%) & $1.3 \pm 0.2$ & $1.3 \pm 0.2$ & $1.3 \pm 0.2$ & 0.436 \\
\hline In-stent lumen expansion * & $85.7 \pm 22.0$ & $88.0 \pm 25.3$ & $85.3 \pm 21.5$ & 0.188 \\
\hline Distal reference lumen area $\left(\mathrm{mm}^{2}\right)$ & $6.4 \pm 2.8$ & $5.7 \pm 2.3$ & $6.4 \pm 2.8$ & 0.002 \\
\hline Proximal reference lumen area $\left(\mathrm{mm}^{2}\right)$ & $8.3 \pm 3.5$ & $7.4 \pm 3.3$ & $8.4 \pm 3.5$ & 0.001 \\
\hline Malapposition thickness (mm) & $0.25 \pm 0.24$ & $0.22 \pm 0.20$ & $0.25 \pm 0.25$ & 0.111 \\
\hline Malapposition length (mm) & $3.3 \pm 4.3$ & $3.0 \pm 3.9$ & $3.4 \pm 4.4$ & 0.259 \\
\hline Intra-stent plaque/thrombus protrusion (mm) & $0.39 \pm 0.25$ & $0.40 \pm 0.28$ & $0.39 \pm 0.25$ & 0.511 \\
\hline Distal edge dissection length $(\mathrm{mm})$ & $0.22 \pm 0.85$ & $0.46 \pm 1.7$ & $0.19 \pm 0.63$ & $<0.001$ \\
\hline Distal edge dissection width (mm) & $0.04 \pm 0.11$ & $0.07 \pm 0.19$ & $0.03 \pm 0.10$ & $<0.001$ \\
\hline Distal edge dissection $\operatorname{arc}\left({ }^{\circ}\right)$ & $6.6 \pm 23.0$ & $14.3 \pm 43.4$ & $5.5 \pm 18.2$ & $<0.001$ \\
\hline Proximal edge dissection length (mm) & $0.14 \pm 0.54$ & $0.20 \pm 0.77$ & $0.13 \pm 0.50$ & 0.183 \\
\hline Proximal edge dissection width (mm) & $0.03 \pm 0.11$ & $0.04 \pm 0.11$ & $0.03 \pm 0.11$ & 0.438 \\
\hline Proximal edge dissection $\operatorname{arc}\left({ }^{\circ}\right)$ & $5.0 \pm 16.7$ & $7.4 \pm 19.9$ & $4.7 \pm 16.2$ & 0.093 \\
\hline \multicolumn{5}{|l|}{ Suboptimal OCT criteria } \\
\hline Minimum in-stent lumen area $<4.5 \mathrm{~mm}^{2}(\%)$ & $338(23.8)$ & $63(35.4)$ & $275(22.1)$ & $<0.001$ \\
\hline Asymmetric stent index $(\%)^{\dagger}$ & $150(10.5)$ & $22(12.4)$ & $128(10.3)$ & 0.479 \\
\hline In-stent lumen underexpansion (\%) $)^{\ddagger}$ & $319(22.4)$ & $46(25.8)$ & $273(21.9)$ & 0.208 \\
\hline Malapposition $>200 \mu(\%)$ & $743(52.3)$ & 87 (48.9) & $656(52.7)$ & 0.337 \\
\hline Intra-stent plaque/thrombus protrusion $>500 \mu(\%)$ & $391(27.5)$ & $53(29.8)$ & $338(27.2)$ & 0.109 \\
\hline Edge dissection $>200 \mu(\%)$ & $170(12.0)$ & $29(16.3)$ & $141(11.3)$ & 0.049 \\
\hline Distal dissection (\%) & $89(6.3)$ & $19(10.7)$ & $70(5.6)$ & 0.007 \\
\hline Proximal dissection (\%) & $92(6.5)$ & $14(7.9)$ & $78(6.3)$ & 0.518 \\
\hline Reference narrowing $(\%)^{\S}$ & $89(6.3)$ & $40(22.5)$ & 49 (3.9) & $<0.001$ \\
\hline Distal narrowing (\%) & $67(4.7)$ & $28(15.7)$ & $39(3.1)$ & $<0.001$ \\
\hline Proximal narrowing (\%) & $29(2.0)$ & $15(8.4)$ & $14(1.1)$ & $<0.001$ \\
\hline
\end{tabular}

* Defined as in-stent to mean reference lumen area, expressed as a percentage. ${ }^{\dagger}$ Ratio between minimum stent diameter/maximum stent diameter $<0.7$. ${ }^{\ddagger}$ Defined as in-stent MLA $<70 \%$ of the average reference lumen area. ${ }^{\S}$ Defined as reference lumen area $<4.5 \mathrm{~mm}^{2}$ in presence of significant plaque.

Table 4. Clinical outcomes.

\begin{tabular}{|c|c|c|c|c|c|c|}
\hline & & $\begin{array}{c}\text { Total population } \\
(1,211)\end{array}$ & $\begin{array}{c}\text { Patients with OCT } \\
\text { suboptimal deployment* } \\
\text { (375) }\end{array}$ & $\begin{array}{c}\text { Patients with OCT } \\
\text { optimal deployment* } \\
\text { (836) }\end{array}$ & $p$-value & HR \\
\hline \multicolumn{2}{|l|}{ DoCE (\%) } & $144(11.9)$ & $76(20.3)$ & $68(8.1)$ & $<0.001$ & $2.70(1.9-3.7)$ \\
\hline \multicolumn{2}{|l|}{ Cardiac death (\%) } & $41(3.4)$ & $19(5.1)$ & $22(2.6)$ & 0.027 & $2.00(1.1-3.7)$ \\
\hline \multicolumn{2}{|l|}{ Target vessel MI (\%) } & $103(7.2)$ & $56(12.7)$ & $47(4.8)$ & $<0.001$ & $2.71(1.8-4.0)$ \\
\hline \multicolumn{2}{|l|}{ Periprocedural } & $42(2.9)$ & $19(4.3)$ & $23(2.3)$ & 0.046 & $1.86(1.1-3.4)$ \\
\hline \multicolumn{2}{|l|}{ During follow-up } & $61(4.3)$ & $37(8.4)$ & $24(2.4)$ & $<0.001$ & $3.46(2.1-5.8)$ \\
\hline \multirow{2}{*}{$\begin{array}{l}\text { Target lesion } \\
\text { revascularisation (\%) }\end{array}$} & patient basis & $102(8.4)$ & $61(16.3)$ & $41(4.9)$ & $<0.001$ & $3.69(2.5-5.5)$ \\
\hline & lesion basis & $122(8.6)$ & 70 (15.9) & $52(5.3)$ & $<0.001$ & $3.22(2.2-4.6)$ \\
\hline \multirow{2}{*}{$\begin{array}{l}\text { Target vessel } \\
\text { revascularisation (\%) }\end{array}$} & patient basis & $124(10.2)$ & $64(17.1)$ & $60(7.2)$ & $<0.001$ & $2.61(1.8-3.7)$ \\
\hline & lesion basis & $152(10.7)$ & $75(17.0)$ & $77(7.8)$ & $<0.001$ & $2.31(1.7-3.2)$ \\
\hline \multirow[t]{2}{*}{ Stent thrombosis (\%) } & patient basis & $32(2.6)$ & $27(7.2)$ & $5(0.6)$ & $<0.001$ & $12.46(4.8-32.3)$ \\
\hline & lesion basis & $34(2.4)$ & $29(6.6)$ & $5(0.5)$ & $<0.001$ & $13.17(5.1-34.0)$ \\
\hline \multicolumn{2}{|l|}{ Days of follow-up ${ }^{\dagger}$} & $833(415-1,447)$ & $746(380-1,458)$ & $881(426-1,445)$ & 0.160 & - \\
\hline
\end{tabular}



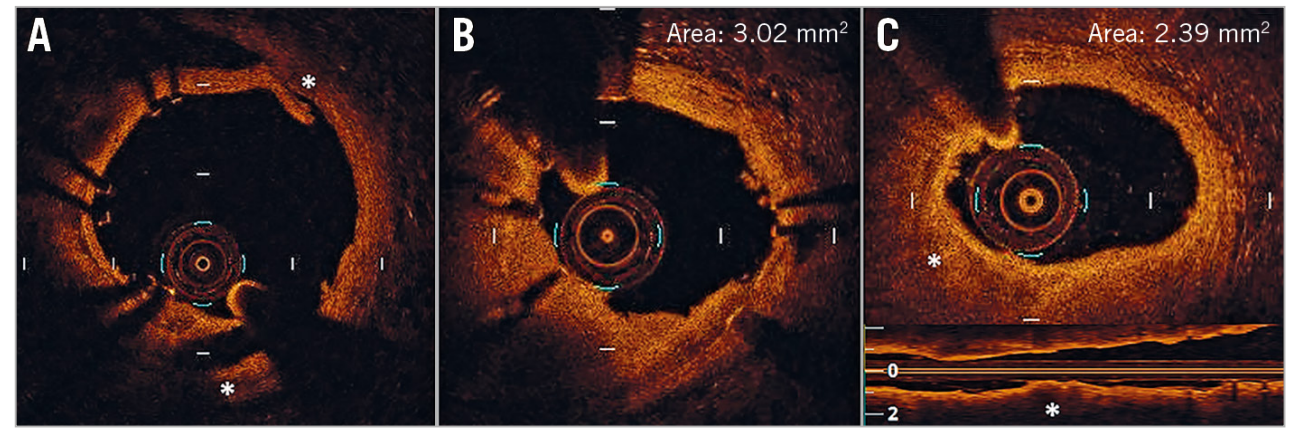

Figure 1. OCT criteria applied to address suboptimal OCT stent deployment. A) Edge dissection (*), defined as a linear rim of tissue with a width $\geq 200 \mu \mathrm{m}$ and a clear separation from the vessel wall or underlying plaque, that was adjacent to a stent edge. B) Intra-stent minimum lumen area $<4.5 \mathrm{~mm}^{2}$. C) Reference lumen narrowing defined as lumen area $<4.5 \mathrm{~mm}^{2}$ in the presence of significant plaque (*) adjacent to stent edges.

plaque/thrombus prolapse $(29.8 \%$ vs. $27.2 \%, \mathrm{p}=0.109)$ were not associated with an increased incidence of adverse events (Table 3).

Cumulatively, the presence of at least one of the predictive OCT parameters (i.e., in-stent MLA $<4.5 \mathrm{~mm}^{2}$, distal stent edge dissection $>200 \mu \mathrm{m}$, and reference narrowing) was disclosed at the end of the procedure in $30.9 \%$ of treated lesions (Table 5) and was significantly more frequent in patients experiencing DoCE during follow-up (48.9\% vs. $28.4 \%, \mathrm{p}<0.001)$. The impact of a suboptimal OCT-defined stent implantation was confined to the early phases of follow-up (HR 2.52, 95\% CI: 1.4-4.6, $\mathrm{p}=0.002$ ), while the clinical outcome was comparable after the first year (HR 1.47, $95 \%$ CI: $0.9-2.5, p=0.165$ ). A Kaplan-Meier curve of the incidence of DoCE is shown in Figure 2.

In the multivariable Cox hazard analysis, suboptimal OCT stent deployment was confirmed as an independent predictor of longterm DoCE (HR 1.92, 95\% CI: 1.3-2.9, p=0.001), together with diabetes mellitus (HR 1.89, 95\% CI: 1.2-2.9, $\mathrm{p}=0.003$ ), chronic kidney disease (HR 1.67, 95\% CI: $1.1-2.7, \mathrm{p}=0.030$ ), and ostial lesion location (HR 2.57, 95\% CI: 1.4-4.7, $\mathrm{p}=0.002$ ).

Table 5. Predictive value of OCT criteria.

\begin{tabular}{|c|c|c|}
\hline $\begin{array}{l}\text { In-stent minimum lumen area } \\
<4.5 \mathrm{~mm}^{2}\end{array}$ & HR $1.82(1.3-2.5)$ & $<0.001$ \\
\hline Eccentricity index $<0.70$ * & HR $1.26(0.8-2.0)$ & 0.320 \\
\hline Stent underexpansion ${ }^{\dagger}$ & HR $1.20(0.9-1.7)$ & 0.292 \\
\hline Malapposition $>200 \mu$ & HR $0.92(0.7-1.2)$ & 0.559 \\
\hline $\begin{array}{l}\text { Intra-stent plaque/thrombus } \\
\text { protrusion }>500 \mu\end{array}$ & HR 1.25 (0.9-1.7) & 0.181 \\
\hline Edge dissection $>200 \mu$ & HR $1.50(1.1-2.2)$ & 0.046 \\
\hline Distal edge dissection $>200 \mu$ & HR 2.03 (1.3-3.3) & 0.004 \\
\hline Proximal edge dissection $>200 \mu$ & HR $1.16(0.7-2.0)$ & 0.587 \\
\hline Reference narrowing ${ }^{\ddagger}$ & HR 5.79 (4.1-8.3) & $<0.001$ \\
\hline Distal reference narrowing ${ }^{\ddagger}$ & HR 5.22 (3.5-7.9) & $<0.001$ \\
\hline Proximal reference narrowing ${ }^{\ddagger}$ & HR 5.67 (3.3-9.7) & $<0.001$ \\
\hline
\end{tabular}

* Ratio between minimum stent diameter/maximum stent diameter. $\dagger$ Defined as in-stent to mean reference lumen area $<70 \%$. $\ddagger$ Defined as reference lumen area $<4.5 \mathrm{~mm}^{2}$ in the presence of significant plaque.

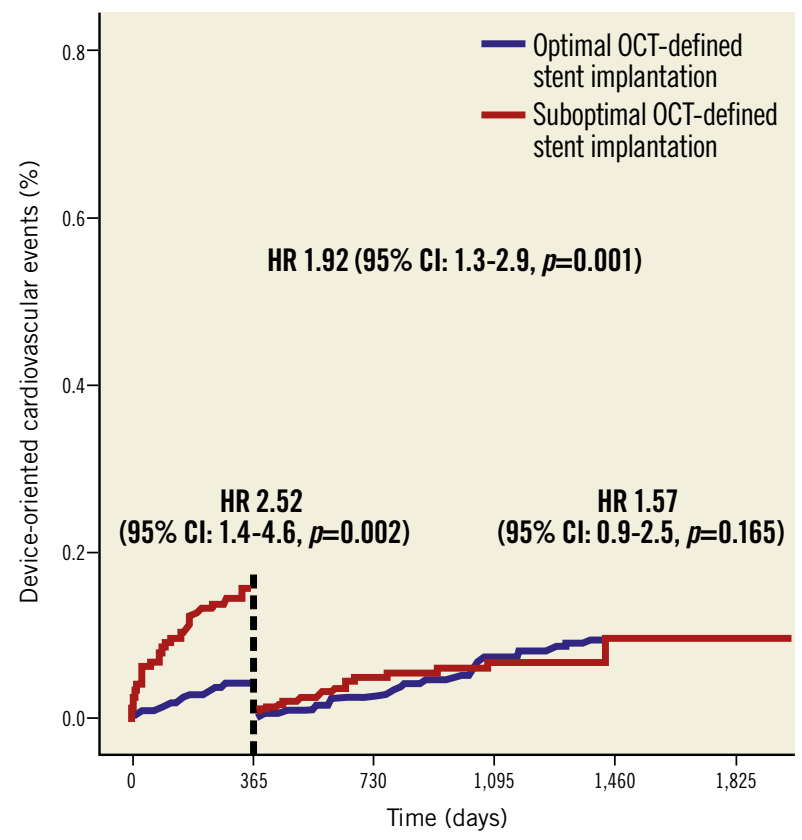

Figure 2. Clinical outcome. Time-to-event curves for device-oriented cardiovascular events (DoCE) according to optimal vs. suboptimal stent deployment assessed using OCT. DoCE: composite of cardiac death, target vessel myocardial infarction, and target lesion revascularisation (TLR)

\section{Discussion}

This study validates, in an adequately large real-world population, the clinical impact of an OCT-defined suboptimal stent implantation at long-term follow-up. In particular, our data confirm the DoCE reduction associated with OCT guidance over standard angiography evaluation during stent implantation, extending to a longer observation period (about three years) the earlier conclusions of the CLI-OPCI project ${ }^{7}$. Interestingly, the same OCT features that were found to be predictive at one-year follow-up remained significantly related to long-term DoCE, with reference narrowing being the metric with by far the highest clinical impact. 


\section{THE CLI-OPCI PROJECT AND EFFECTIVE METRICS OF SUBOPTIMAL STENTING}

The CLI-OPCI project was designed with the goal of systematically collecting from high-volume OCT-experienced centres data on all consecutive PCIs performed with intraprocedural OCT assessment. The clinical usefulness of OCT-specific metrics was originally tested in the CLI-OPCI registry (335 patients) that showed a clinical benefit at one year in terms of cardiac death and myocardial infarction with OCT guidance ${ }^{6}$. Subsequently, the larger CLI-OPCI II study (832 patients) identified OCT metrics of suboptimal stent deployment (in-stent MLA $<4.5 \mathrm{~mm}^{2}$, dissection $>200 \mu \mathrm{m}$ at the distal stent edge, and reference lumen area $<4.5 \mathrm{~mm}^{2}$ in the presence of residual significant plaque at stent edges) as being associated with worse outcome in the first year of follow-up ${ }^{7}$.

The current CLI-OPCI LATE study enrolling a total of 1,211 patients with a median follow-up of almost three years aimed to evaluate the clinical benefit of an optimal OCT-defined stent implantation at long-term follow-up. A cumulative DoCE rate of $11.9 \%$ was recorded with an incidence of cardiac death $(3.4 \%)$, target vessel-related MI (7.2\%), and TLR (8.6\%) consistent with other large all-comer PCI registries ${ }^{19}$. Despite the satisfactory angiographic result, the end-procedural OCT assessment revealed a significant rate of suboptimal stent deployment $(30.9 \%$ of lesions) that was significantly higher in patients experiencing DoCE $(48.9 \%$ vs. $28.4 \%, p<0.001)$. In fact, patients with at least one of the predictive OCT criteria showed an increased risk of cardiac death (HR 2.0), target vessel MI (HR 1.86 for periprocedural and HR 3.46 for post-discharge MI), and TLR (HR 3.22). After correction for other potential confounding factors and comorbidities, the presence of a final suboptimal stent deployment was confirmed as an independent outcome predictor (HR 1.92). Notably, all predictive OCT metrics identified in the CLI-OPCI II - in-stent MLA $<4.5 \mathrm{~mm}^{2}$, dissection $>200 \mu \mathrm{m}$ at the distal stent edge, and reference vessel disease with lumen area $<4.5 \mathrm{~mm}^{2}$ - remained associated with a worse outcome, albeit their predictive strength was maximal during the first year of follow-up (Table 5, Figure 2). The observed attenuation of the OCT clinical impact with a stabilisation of survival curve divergence after the first year probably reflects the emerging influence of the baseline clinical risk profile and worsening of atherosclerosis that became more evident at long-term follow-up.

\section{NON-EFFECTIVE OCT METRICS OF SUBOPTIMAL STENTING}

According to the one-year data of previous CLI-OPCI regis$\operatorname{tries}^{6-8}$, and also as seen in the present study, acute residual SM, variably observed in over $50 \%$ of stents, was not significantly related to the risk of long-term stent failure (HR 0.92). Albeit the lack of an OCT follow-up is a limitation in discussing the role of acute SM as a potential cause of stent thrombosis due to incomplete stent coverage ${ }^{20,21}$, the present OCT findings (consistent with the ADAPT-DES IVUS data ${ }^{22}$ ), seem to suggest a safe management of acute residual SM with single antiplatelet therapy after the first year ${ }^{23}$.
Similarly, \% stent narrowing (i.e., relative stent underexpansion), considered the most important metric to address the adequacy of stent deployment in most randomised studies ${ }^{24,25}$, was not related to a worse clinical outcome in the current analysis (HR 1.20). This finding, probably due to lumen/calibre mismatch between diseased and healthy reference segments, should be considered when designing future randomised studies in order to assess the clinical utility of OCT guidance.

\section{Limitations}

The retrospective design represents the main limitation of the present study. Indeed, this registry included patients with different clinical conditions uniquely pooled by intraprocedural OCT use (not randomised). Nevertheless, after correction for the evident clinical and procedural differences, the presence of non-optimal OCT criteria for stent deployment was confirmed as an independent predictor of DoCE in the multivariable Cox hazard analysis.

Starting from the generally adopted IVUS/OCT definitions of suboptimal stent deployment, in the present study, and in the CLI-OPCI registries ${ }^{6-8}$, we proposed luminal cut-offs to delineate a practical approach to OCT guidance. However, this approach needs further clinical validation from randomised studies as the role and importance of the described OCT findings could vary according to different patient/device categories.

\section{Conclusions}

The presence of a suboptimal stent deployment, defined according to specific quantitative OCT criteria, was confirmed as an independent outcome predictor at long-term follow-up. These data add more evidence to the clinical utility of an OCT-guided strategy during PCI.

\section{Impact on daily practice}

In the context of a large multicentre real-world registry (the Centro per la Lotta Contro l'Infarto - Optimisation of Percutaneous Coronary Intervention project), this study demonstrated a correlation between optical coherence tomography (OCT)-defined suboptimal stent deployment and worse longterm clinical outcome. In particular, the presence of distal stent edge dissection $>200 \mu \mathrm{m}$, in-stent minimum lumen area $<4.5 \mathrm{~mm}^{2}$, and significant reference vessel plaque with a lumen area $<4.5 \mathrm{~mm}^{2}$ at the stent edges, are independent predictors of device failure. Larger randomised prospective studies are needed to confirm the clinical impact of OCT guidance during percutaneous coronary intervention and to investigate the management and the possibility of improvement of OCT-defined suboptimal stent deployment.

\section{Appendix. CLI-OPCI project study investigators}

Executive committee: Francesco Prati, MD (principal investigator and chair), Ospedale San Giovanni - Addolorata, Rome, Italy; Gary S. Mintz, MD, Cardiovascular Research Foundation, New York, 
NY, USA; Fernando Alfonso, MD, Hospital Universitario de la Princesa, IIS-IP, Madrid, Spain.

Steering committee: Corrado Tamburino, MD, Azienda Ospedaliera Universitaria Ferrarotto, Catania, Italy; Francesco Burzotta, MD, PhD, Università Cattolica Del Sacro Cuore, Rome, Italy; Enrico Romagnoli, $\mathrm{MD}, \mathrm{PhD}$, Ospedale San Giovanni Addolorata, Rome, Italy; Laura Gatto, MD, Ospedale San Giovanni - Addolorata, Rome, Italy; Ugo Limbruno, MD, Ospedale della Misericordia, Grosseto, Italy; Eloisa Arbustini, MD, Fondazione IRCCS Policlinico San Matteo, Pavia, Italy.

Clinical sites and investigators: Valeria Marco, RT, Centro per la Lotta Contro L'Infarto, Rome, Italy; Chiara Russo, RT, Centro per la Lotta Contro L'Infarto, Rome, Italy; Alessio la Manna, MD, Azienda Ospedaliera Universitaria Ferrarotto, Catania, Italy; Giovanni Ruscica, MD, Azienda Ospedaliera Universitaria Ferrarotto, Catania, Italy; Italo Porto, MD, $\mathrm{PhD}$, Università Cattolica Del Sacro Cuore, Rome, Italy; Giampaolo Niccoli, $\mathrm{MD}, \mathrm{PhD}$, Università Cattolica Del Sacro Cuore, Rome, Italy; Carlo Trani, MD, Università Cattolica Del Sacro Cuore, Rome, Italy; Filippo Crea, MD, PhD, Università Cattolica Del Sacro Cuore, Rome, Italy; Nevio Taglieri, MD, Policlinico Sant'Orsola-Malpighi, Bologna, Italy; Francesco Saia, MD, Policlinico Sant'Orsola-Malpighi, Bologna, Italy; Silvio Fedele, MD, Azienda Ospedaliera Sandro Pertini, Rome, Italy; Francesco Versaci, MD, Ospedale Santa Maria Goretti, Latina, Italy; Francesco Amico, MD, Presidio Ospedaliero Sant'Elia, Caltanissetta, Italy; Salvatore Azzarelli, MD, Presidio Ospedaliero Sant'Elia, Caltanissetta, Italy; Vito Ramazzotti, MD, Ospedale San Giovanni - Addolorata, Rome, Italy; Alessandro Manzoli, MD, Ospedale San Giovanni - Addolorata, Rome, Italy; Fabrizio Imola, MD, Ospedale San Giovanni - Addolorata, Rome, Italy; Alessandro Pappalardo, MD, Ospedale San Giovanni - Addolorata, Rome, Italy; Mario Albertucci, MD, Ospedale San Giovanni - Addolorata, Rome, Italy; Franco Fabbiocchi, MD, Centro Cardiologico Monzino IRCCS, Milan, Italy; Giuseppe Z. Calligaris, MD, Centro Cardiologico Monzino IRCCS, Milan, Italy; Luca Di Vito, MD, PhD, Azienda Ospedaliera Mazzoni, Ascoli Piceno, Italy; Alessandro Di Giorgio, MD, Policlinico G. Martino, Messina, Italy; Antonio Bracco, MD, Policlinico G. Martino, Messina, Italy; Alberto Boi, MD, Ospedale Brotzu, Cagliari, Italy; Angelica Rossi, MD, Ospedale Brotzu, Cagliari, Italy; Marco Contarini, MD, Presidio Ospedaliero Umberto I, Syracuse, Italy; Giorgio Sacchetta, MD, Presidio Ospedaliero Umberto I, Syracuse, Italy; Antonio Trivisonno, MD, Ospedale A. Cardarelli, Campobasso, Italy; Irene Pescetelli, MD, Ospedale A. Cardarelli, Campobasso, Italy; Andrea Picchi, MD, Ospedale della Misericordia, Grosseto, Italy; Paolo Calabria, MD, Ospedale della Misericordia, Grosseto, Italy; Massimo Fineschi, MD, Azienda Ospedaliera Universitaria Senese, Siena, Italy; Elisabetta Iardino, MD, Azienda Ospedaliera Universitaria Senese, Siena, Italy.

\section{Funding}

The CLI-OPCI project has been entirely sponsored by the Centro per la Lotta Contro l'Infarto - Fondazione Onlus, Rome, Italy, with no extramural funding.

\section{Conflict of interest statement}

F. Prati has served as a consultant for St. Jude Medical. G. Mintz has served as a consultant for or has received honoraria from Boston Scientific, Volcano, ACIST, and Infraredx. F. Burzotta has received speaker's fees from Medtronic, Abiomed, and St. Jude. The other authors have no conflicts of interest to declare.

\section{References}

1. Prati F, Guagliumi G, Mintz GS, Costa M, Regar E, Akasaka T, Barlis P, Tearney GJ, Jang IK, Arbustini E, Bezerra HG, Ozaki Y, Bruining N, Dudek D, Radu M, Erglis A, Motreff P, Alfonso F, Toutouzas K, Gonzalo N, Tamburino C, Adriaenssens T, Pinto F, Serruys PW, Di Mario C; Expert's OCT Review Document. Expert review document part 2: methodology, terminology and clinical applications of optical coherence tomography for the assessment of interventional procedures. Eur Heart J. 2012;33:2513-20.

2. Ahn JM, Kang SJ, Yoon SH, Park HW, Kang SM, Lee JY, Lee SW, Kim YH, Lee CW, Park SW, Mintz GS, Park SJ. Metaanalysis of outcomes after intravascular ultrasound-guided versus angiography-guided drug-eluting stent implantation in 26,503 patients enrolled in three randomized trials and 14 observational studies. Am J Cardiol. 2014;113:1338-47.

3. Witzenbichler B, Maehara A, Weisz G, Neumann FJ, Rinaldi MJ, Metzger DC, Henry TD, Cox DA, Duffy PL, Brodie BR, Stuckey TD, Mazzaferri EL Jr, Xu K, Parise H, Mehran R, Mintz GS, Stone GW. Relationship between intravascular ultrasound guidance and clinical outcomes after drug-eluting stents: the assessment of dual antiplatelet therapy with drug-eluting stents (ADAPT-DES) study. Circulation. 2014;129: 463-70.

4. Soeda T, Uemura S, Park SJ, Jang Y, Lee S, Cho JM, Kim SJ, Vergallo R, Minami Y, Ong DS, Gao L, Lee H, Zhang S, Yu B, Saito Y, Jang IK. Incidence and Clinical Significance of Poststent Optical Coherence Tomography Findings: One-Year Follow-Up Study From a Multicenter Registry. Circulation. 2015;132:1020-9.

5. Mintz GS. Clinical utility of intravascular imaging and physiology in coronary artery disease. J Am Coll Cardiol. 2014;64:207-22.

6. Prati F, Di Vito L, Biondi-Zoccai G, Occhipinti M, La Manna A, Tamburino C, Burzotta F, Trani C, Porto I, Ramazzotti V, Imola F, Manzoli A, Materia L, Cremonesi A, Albertucci M. Angiography alone versus angiography plus optical coherence tomography to guide decision-making during percutaneous coronary intervention: the Centro per la Lotta contro l'InfartoOptimisation of Percutaneous Coronary Intervention (CLI-OPCI) study. EuroIntervention. 2012;8:823-9.

7. Prati F, Romagnoli E, Burzotta F, Limbruno U, Gatto L, La Manna A, Versaci F, Marco V, Di Vito L, Imola F, Paoletti G, Trani C, Tamburino C, Tavazzi L, Mintz GS. Clinical Impact of OCT Findings During PCI: The CLI-OPCI II Study. JACC Cardiovasc Imaging. 2015;8:1297-305.

8. Prati F, Romagnoli E, Gatto L, La Manna A, Burzotta F, Limbruno U, Versaci F, Fabbiocchi F, Di Giorgio A, Marco V, 
Ramazzotti V, Di Vito L, Trani C, Porto I, Boi A, Tavazzi L, Mintz GS. Clinical Impact of Suboptimal Stenting and Residual Intrastent Plaque/Thrombus Protrusion in Patients With Acute Coronary Syndrome: The CLI-OPCI ACS Substudy (Centro per la Lotta Contro L'Infarto-Optimization of Percutaneous Coronary Intervention in Acute Coronary Syndrome). Circ Cardiovasc Interv. 2016 Dec;9(12).

9. Imola F, Mallus MT, Ramazzotti V, Manzoli A, Pappalardo A, Di Giorgio A, Albertucci M, Prati F. Safety and feasibility of frequency domain optical coherence tomography to guide decision making in percutaneous coronary intervention. EuroIntervention. 2010;6:575-81.

10. Prati F, Cera M, Ramazzotti V, Imola F, Giudice R, Giudice M, Propris SD, Albertucci M. From bench to bedside: a novel technique of acquiring OCT images. Circ J. 2008;72:839-43.

11. Thygesen K, Alpert JS, Jaffe AS, Simoons ML, Chaitman BR, White HD; Writing Group on the Joint ESC/ACCF/AHA/WHF Task Force for the Universal Definition of Myocardial Infarction; ESC Committee for Practice Guidelines (CPG). Third universal definition of myocardial infarction. Eur Heart J. 2012;33: 2551-67.

12. Cutlip DE, Windecker S, Mehran R, Boam A, Cohen DJ, van Es GA, Steg PG, Morel MA, Mauri L, Vranckx P, McFadden E, Lansky A, Hamon M, Krucoff MW, Serruys PW; Academic Research Consortium. Clinical end points in coronary stent trials: a case for standardized definitions. Circulation. 2007;115: 2344-51

13. Popma JJ, Bashore TD. Qualitative and quantitative angiography. In: Topol E, editor. Textbook of Interventional Cardiology. Philadelphia, PA, USA: WB Saunders; 1994. p.1052-68.

14. Tanigawa J, Barlis P, Di Mario C. Intravascular optical coherence tomography: optimization of image acquisition and quantitative assessment of stent strut apposition. EuroIntervention. 2007; 3:128-36

15. Egan JP. Signal detection theory and ROC analysis. New York, NY, USA: Academic Press; 1975.

16. Youden WJ. An index for rating diagnostic tests. Cancer. 1950;3:32-5.

17. Prati F, Regar E, Mintz GS, Arbustini E, Di Mario C, Jang IK, Akasaka T, Costa M, Guagliumi G, Grube E, Ozaki Y, Pinto F, Serruys PW; Expert's OCT Review Document. Expert review document on methodology, terminology, and clinical applications of optical coherence tomography: physical principles, methodology of image acquisition, and clinical application for assessment of coronary arteries and atherosclerosis. Eur Heart J. 2010;31:401-15.

18. Kume T, Akasaka T, Kawamoto T, Ogasawara Y, Watanabe N, Toyota E, Neishi Y, Sukmawan R, Sadahira Y, Yoshida K.
Assessment of coronary arterial thrombus by optical coherence tomography. Am J Cardiol. 2006;97:1713-7.

19. Meredith I, Rothman M, Erglis A, Parikh K, Lotan C; E-Five Investigators. Extended follow-up safety and effectiveness of the Endeavor zotarolimus-eluting stent in real-world clinical practice: two-year follow-up from the E-Five Registry. Catheter Cardiovasc Interv. 2011;77:993-1000.

20. Guagliumi G, Sirbu V, Musumeci G, Gerber R, BiondiZoccai G, Ikejima H, Ladich E, Lortkipanidze N, Matiashvili A, Valsecchi O, Virmani R, Stone GW. Examination of the in vivo mechanisms of late drug-eluting stent thrombosis: findings from optical coherence tomography and intravascular ultrasound imaging. JACC Cardiovasc Interv. 2012;5:12-20.

21. Taniwaki M, Radu MD, Zaugg S, Amabile N, GarciaGarcia HM, Yamaji K, Jørgensen E, Kelbæk H, Pilgrim T, Caussin C, Zanchin T, Veugeois A, Abildgaard U, Jüni P, Cook S, Koskinas KC, Windecker S, Räber L. Mechanisms of Very Late Drug-Eluting Stent Thrombosis Assessed by Optical Coherence Tomography. Circulation. 2016;133:650-60.

22. Wang B, Mintz GS, Witzenbichler B, Souza CF, Metzger DC, Rinaldi MJ, Duffy PL, Weisz G, Stuckey TD, Brodie BR, Matsumura M, Yamamoto MH, Parvataneni R, Kirtane AJ, Stone GW, Maehara A. Predictors and Long-Term Clinical Impact of Acute Stent Malapposition: An Assessment of Dual Antiplatelet Therapy With Drug-Eluting Stents (ADAPT-DES) Intravascular Ultrasound Substudy. J Am Heart Assoc. 2016 Dec 22;5(12).

23. Romagnoli E, Gatto L, La Manna A, Burzotta F, Taglieri N, Saia F, Amico F, Marco V, Ramazzotti V, Di Giorgio A, Di Vito L, Boi A, Contarini M, Castriota F, Mintz GS, Prati F. Role of residual acute stent malapposition in percutaneous coronary interventions. Catheter Cardiovasc Interv. 2017;90:566-75.

24. Ali ZA, Maehara A, Généreux P, Shlofmitz RA, Fabbiocchi F, Nazif TM, Guagliumi G, Meraj PM, Alfonso F, Samady H, Akasaka T, Carlson EB, Leesar MA, Matsumura M, Ozan MO, Mintz GS, Ben-Yehuda O, Stone GW; ILUMIEN III: OPTIMIZE PCI Investigators. Optical coherence tomography compared with intravascular ultrasound and with angiography to guide coronary stent implantation (ILUMIEN III: OPTIMIZE PCI): a randomised controlled trial. Lancet. 2016;388:2618-28.

25. Meneveau N, Souteyrand G, Motreff P, Caussin C, Amabile N, Ohlmann P, Morel O, Lefrançois Y, Descotes-Genon V, Silvain J, Braik N, Chopard R, Chatot M, Ecarnot F, Tauzin H, Van Belle E, Belle L, Schiele F. Optical Coherence Tomography to Optimize Results of Percutaneous Coronary Intervention in Patients with NonST-Elevation Acute Coronary Syndrome: Results of the Multicenter, Randomized DOCTORS Study (Does Optical Coherence Tomography Optimize Results of Stenting). Circulation. 2016;134:906-17. 